\title{
Continuous Object Monitoring Based on Wireless Sensor Network and RBF
}

\author{
Chengping Peng ${ }^{a}$, Guangcong Liu ${ }^{b}$ \\ School of Computer, Guangdong University of Technology, Guangzhou 510000, China; \\ apechpi@163.com, bliugc@gdut.edu.cn
}

\begin{abstract}
Keywords: Gaussian smoke model, wireless sensor network, continuous object, RBF neural network, monitoring.
\end{abstract}

\begin{abstract}
Monitoring of continuous object distribution in large-scale environmental monitoring depends on the problem of high-density sensor networks. The RBF neural network is used to fit the distributed data of continuous objects at low sensor network density. Firstly, based on the distribution characteristics of continuous objects, a continuous evolution model of continuous objects based on Gaussian smoke model [1] is established. Secondly, the RBF neural network is trained based on the known distributed node data. Thirdly, RBF neural network is used to fit the distribution data of continuous objects. Finally, the fitting error of different number of training nodes is calculated. Through the experiment of matlab simulation, the practicability of RBF neural network for continuous object distribution has been validated.
\end{abstract}

\section{Introduction}

With the rapid development of hardware and software, embedded and the Internet, wireless sensor networks ${ }^{[2-3]}$ quickly applied in the all aspects of life. Especially in the areas of personal safety, such as forest fire prevention, toxic and harmful gas monitoring, earthquakes, tsunamis and so on. And the use of large-scale wireless sensor network can accurately monitor these states, and give people enough time to prepare to deal with the crisis. When these states are studied, these objects are generally looked at continuous objects. Continuous objects ${ }^{[4-6]}$ generally refers to the volume and shape can not be ignored, cannot be regarded as the object of particles. The main task of monitoring the continuous object is to monitor the boundary of the continuous object. The essence of the problem is to use the discrete point of the wireless sensor to restore the continuous object attribute.

At present, most of the researches on wireless sensor networks are boundary detection and location, and the research on continuous object ${ }^{[7-9]}$ monitoring by wireless sensor network is relatively few. In the traditional continuous object monitoring, in order to improve the monitoring accuracy, the general deployment of large-scale sensors. Compared with traditional methods of monitoring continuous objects by large-scale deployment of sensors, the content of this paper is to reduce the number of sensors, which should be employed to monitor continuous objects as few sensors as possible.

\section{Models and algorithms}

\subsection{Gaussian Smoke Diffusion Model}

Under the premise of considering plane diffusion only, the Gaussian smoke diffusion model is adopted:

$$
c(x, y)=\frac{2 Q}{(2 \pi)^{3 / 2} \delta_{x} \delta_{y}} \exp \left(\frac{-x^{2}}{2 \delta_{x}{ }^{2}}\right) \exp \left(\frac{-y^{2}}{2 \delta_{y}{ }^{2}}\right)
$$

Where $\mathrm{Q}$ is the gas source intensity, $\delta_{x}$ and $\delta_{y}$ are the $\mathrm{x}$ and y direction diffusion parameters, respectively. The dynamic evolution of continuous objects can be considered to be the result of multiple groups of cigarettes spreading over time, weakening, translating and superimposing. Therefore, at time $t$, the distribution state $\mathrm{D}(\mathrm{t})$ of the continuous object is: 


$$
\begin{aligned}
& D(t)=\sum_{i=0}^{t} c(x, y)_{i} \\
& c(x, y)_{i}=\frac{2 Q_{i}}{(2 \pi)^{3 / 2} \delta_{x i} \delta_{y i}} \exp \left(\frac{-\left(x-u \times i \times \operatorname{direc}(i)_{x}\right)^{2}}{2 \delta_{x i}{ }^{2}}\right) \exp \left(\frac{-\left(y-u \times i \times \operatorname{direc}(i)_{y}\right)^{2}}{2 \delta_{y i}{ }^{2}}\right) \\
& Q_{i}=Q_{i-1}-\partial \times T \\
& \delta_{x, i}=\delta_{x, i-1}+\beta \times T \\
& \delta_{y, i}=\delta_{y, i-1}+\beta \times T
\end{aligned}
$$

Where $\mathrm{i}$ is the diffusion time, and $\mathrm{d}$ is the diffusion direction, $\mathrm{d}$ (i) $\mathrm{x}$ and $\mathrm{d}$ (i) $\mathrm{y}$ are the diffusion direction signs in the $\mathrm{x}$ and $\mathrm{y}$ directions at time $\mathrm{i}$, respectively, when in the opposite direction, they are netagive values. The intensity of the smoke center at time i, $\delta_{x, i}$ and $\delta_{y, i}$ are the diffusion coefficients of the smoke group in the $\mathrm{x}$ and $\mathrm{y}$ directions, respectively. $\delta$ and $\beta$ are the weakening and diffusion intensity coefficients of the smoke group. In the dynamic evolution of continuous objects, the concentration distribution and the distance have the trigonometric function, linear and normal distribution.

\subsection{The continuous object fitting corresponds to the RBF network model}

The diffusion and evolution of continuous objects are geometrically distributed. The input of RBF neural network is the distance vector of the sensor fitting point to the reference point, and the output is the sensor sampling data. Continuous object sample training is defined as follows:

$$
\begin{aligned}
& I=\left[\begin{array}{ccccc}
0 & d_{12} & d_{13} & \cdots & d_{1 N} \\
d_{21} & 0 & d_{23} & \cdots & d_{2 N} \\
d_{31} & d_{32} & 0 & \cdots & d_{3 N} \\
\vdots & \vdots & \vdots & \cdots & \vdots \\
d_{N 1} & d_{N 2} & d_{N 3} & \cdots & 0
\end{array}\right] \\
& \boldsymbol{O}=\left[\begin{array}{lllll}
v_{1} & v_{2} & v_{3} & \cdots & v_{N}
\end{array}\right]
\end{aligned}
$$

among them, $d_{i, j}=\sqrt{\left(x_{i}-x_{j}\right)^{2}+\left(y_{i}-y_{j}\right)^{2}} \mathrm{Vi}$ is the sampled data of sensor $\mathrm{i}$, the structure of RBF neural network is as follows:

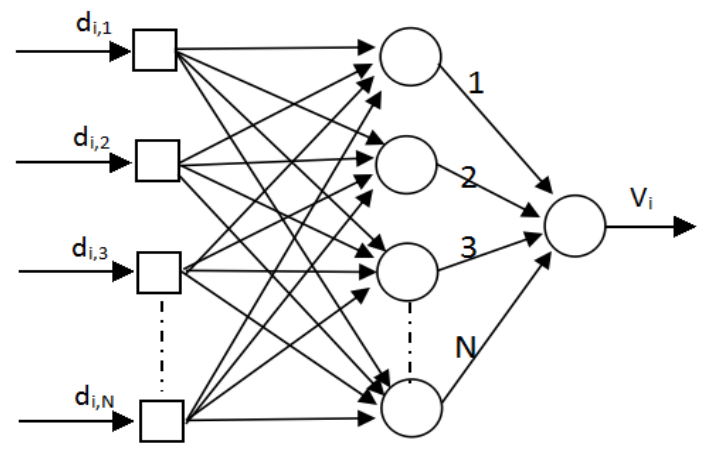

Figure $1 \mathrm{RBF}$ network model

\subsection{Algorithm Description}

(1) Using Matlab for simulation, The machine deploys the sensor to the grid division area, and each grid has at most one sensor node.

(2) Collect sensor network data and train RBF neural network.

(3) The RBF neural network is used to fit the whole region.

(4) The optimal number of nodes is determined according to the different training samples and the gradient graph of the continuous object distribution is drawn. 


\section{Experimental results and analysis}

\subsection{Experimental environment and equipment}

Table 1. Experimental environment and equipment

\begin{tabular}{cc}
\hline Experimental parameters & Value \\
\hline RBF neural network function & newrb(P,T,goal,spreadc,MN,DF $)$ \\
RBF neural network function & newrbe(P,T,spread) \\
experiment platform & Matlab2014b \\
Experiment environment & Windows8.1 4core \\
\hline
\end{tabular}
Value

\subsection{Continuous Object Distribution Fitting Based on RBF Neural Network}

On the basis of the Gaussian Smoke model, it is assumed that the target range is 100X100 unit area. The sensor nodes were randomly distributed in the 10,000 unit areas, and then the RBF neural network was established to fit the 10,000 unit area. So you can create N input, 1 output RBF network. Define the sensor network absolute fit error D as follows:

$$
\mathrm{D}=\sqrt{(M t-M s)^{2}} / 10000
$$

Where Mt is the actual value and Ms is the fitting value.

In the matlab neural network toolbox, The main functions for RBF neural network design are newrb and newrbe. Through the experiment, the number of sensor network nodes is 50 to 300 steps of 50 newrb and newrbe training time as follows:
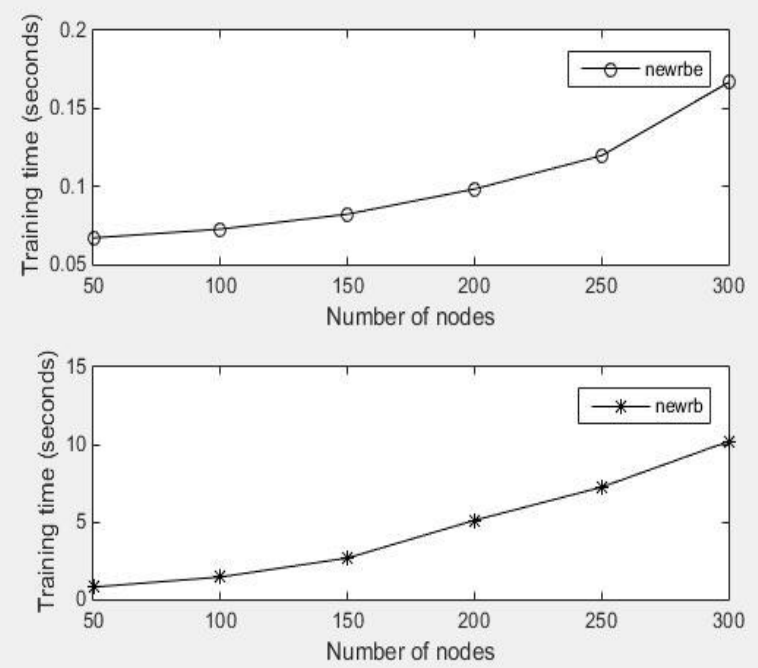

Figure 2 newrbe and newrb fitting time

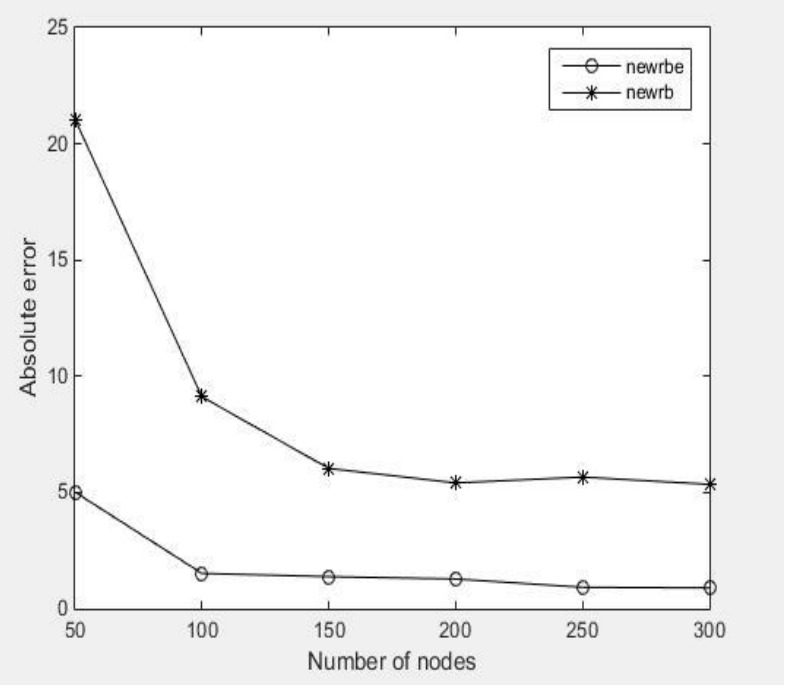

Figure 3 Error comparison

From Figure 2, with the increase in the number of sample nodes, newrb and newrbe training time are correspondingly increased. But newrb training time to be higher anrr an order of magnitude. As can be seen from Figure 3, with the increase of the sample nodes, the absolute difference is reduced and the absolute error of newrb is always higher than newrbe. When the sample node to about 150 , then increase the absolute error of the sample node is basically unchanged.

\section{Summary}

In this paper, we study the continuous object monitoring in large-scale environmental monitoring. The distribution characteristics of continuous objects are analyzed, and the dynamic evolution model of continuous objects based on Gaussian smoke model is established. The influence of RBF network structure on monitoring performance is analyzed experimentally.

Although the experimental process of this paper is based on the continuous object distribution simulation with the Gaussian Smoke model, the experimental conclusion has some limitations, but the continuous distribution of the characteristic parameters of the continuous object is characterized by the 
continuity of the continuous object monitoring process. This paper does not use the established mathematical formula to fit the target object, and the artificial intelligence algorithm based on RBF network is used to fit the continuous object. This makes full use of the real-time and dynamic performance of the sensor network data acquisition, which makes the continuous. The method of object distribution parameter monitoring can adapt to complex and variable continuous object monitoring tasks.

\section{References}

[1] Chen J, Salim M B, Matsumoto M. A Gaussian Mixture Model-Based Continuous Boundary Detection for 3D Sensor Networks[J]. Sensors, 2010, 10(8): 7632-7650. [9]. Yang C, Li Q, Liu J. A multisink-based Continuous Object Tracking in wireless sensor networks by GIS[C]. Advanced Communication Technology (ICACT), 2012 14th International Conference on. IEEE, 2012: 7-11.

[2] Sun Limin, Li Jianzhong, Chen Yu, etc .. Wireless sensor network [M]. Tsinghua University Press. 2005.5: 148-154.

[3] Cheng W, Zhang N, Cheng X, et al. Time-bounded essential localization for wireless sensor networks[J]. IEEE/ACM Transactions on Networking (TON), 2013, 21(2): 400-412.

[4] Hong H, Oh S, Lee J, et al. A Chaining Selective Wakeup Strategy for a Robust Continuous Object Tracking in Practical Wireless Sensor Networks[C]. Advanced Information Networking and Applications (AINA), 2013 IEEE 27th International Conference on. IEEE, 2013: 333-339.

[5] Hong S W, Noh S K, Ryu H Y, et al. A Novel Continuous Object Tracking Scheme for Energy-Constrained Wireless Sensor Networks[C]. Vehicular Technology Conference Fall (VTC 2010-Fall), 2010 IEEE 72nd. IEEE, 2010: 1-5.

[6] Lee W, Yim Y, Park S, et al. A Cluster-based Continuous Object Tracking Scheme in Wireless Sensor Networks[C]. Vehicular Technology Conference (VTC Fall), 2011 IEEE. IEEE, 2011: 1-5. [33].

[7] Park B, Park S, Lee E, et al. Detection and Tracking of Continuous Objects for Flexibility and Reliability in Sensor Networks[C]. Communications (ICC), 2010:1-6.

[8] Chauhdary S H, Lee J, Shah S C, et al. EBCO-Efficient Boundary Detection and Tracking Continuous Objects in WSNs[J]. KSII Transactions on Internet and Information Systems (TIIS), 2012, 6(11): 2901-2919.

[9] Hong S W, Noh S K, Lee E, et al. Energy-efficient predictive tracking for continuous objects in wireless sensor networks[C]. Personal Indoor and Mobile Radio Communications (PIMRC), 2010 IEEE 21st International Symposium on. IEEE, 2010: 1725-1730. 